\title{
Correction to: Enhancers and Promoters
}

\section{Tilman Borggrefe and Benedetto Daniele Giaimo}

\section{Correction to:}

Chapters 4 and 11 in: Tilman Borggrefe and Benedetto Daniele Giaimo (eds.)), Enhancers and Promoters: Methods and Protocols, Methods in Molecular Biology, vol. 2351, https://doi.org/10.1007/978-1-0716-1597-3_21

Chapters 4 and 11 were previously published non-open access. They have now been changed to open access under a CC BY 4.0 license and the copyright holder updated to 'The Author(s)'. The book has been updated with these changes. 